\title{
Actitudes, percepciones de autoeficacia y prácticas de docentes de preescolar hacia el trabajo con familias homoparentales en Bogotá
}

\section{Preschool teachers' attitudes, self-efficacy perceptions and practices towards working with same-sex parent families in Bogotá}

\author{
Carlos Hermosa-Bosano \\ Universidad de Las Américas, Quito, Ecuador- Universidad de los Andes. Bogotá, Colombia. \\ Elvia Vargas-Trujillo \\ Universidad de los Andes, Bogotá, Colombia \\ Karen Ripoll-Núñez \\ Universidad de los Andes, Bogotá, Colombia
}

(Rec: febrero de 2019 - Acept: octubre de 2019)

\begin{abstract}
Resumen
Menos del $30 \%$ de la población en Colombia está de acuerdo con que las parejas del mismo sexo adopten. Este clima de rechazo trae interrogantes sobre las experiencias de las familias homoparentales en el país. Debido a la importancia de los años preescolares para el desarrollo de niñas y niños, este estudio tuvo como objetivo describir las actitudes, percepciones de autoeficacia y prácticas de 53 docentes de prescolar en Bogotá, con relación al trabajo conjunto con los distintos miembros de familias homoparentales. El análisis de las encuestas revela preocupación ante la potencial victimización a la que pueden estar expuestas las hijas e hijos de estas familias. Así mismo, se encontró que, si bien afirman sentirse competentes, las y los docentes reconocen que con baja frecuencia implementan estrategias para construir climas escolares inclusivos. Los resultados permiten recomendar el desarrollo de iniciativas de formación para docentes para que, desde los primeros años de educación formal, los miembros de familias homoparentales cuenten con entornos afirmativos de la diversidad.
\end{abstract}

Palabras claves: Familias homoparentales, educación preescolar, docentes, diversidad

\begin{abstract}
Less than $30 \%$ of the population in Colombia agrees with same-sex couples adopting children. Given this climate of rejection, it is important to answer questions regarding same-sex parent families' experiences with systems such as schools. Due to the importance of preschool years in children's development, this study aimed to describe the attitudes, self-efficacy perceptions and practices of 53 preschool teachers in Bogotá towards working with same-sex parent families. The data analysis revealed concerns regarding the potential victimization that might affect children given their family structure. Furthermore, although teachers reported feeling mostly confident, it was found that they rarely use strategies to build safe and inclusive school environments. These results highlight the importance of working with teachers to create inclusive environments towards same-sex parent families from the very first years of compulsory education. Keywords: Same-sex parent families, preschool education, teachers, diversity
\end{abstract}




\section{Introducción}

A pesar de que cada vez existe mayor aceptación hacia las personas lesbianas. gais y bisexuales (LGB) . existe todavía rechazo hacia la posibilidad de que adopten y construyan familias (Newton Webb \& Chonody. 2014). En Colombia. los datos de la última Encuesta Nacional de Demografía y Salud (ENDS) confirman esta tendencia y revelan que más del $80 \%$ de la población considera que las personas homosexuales deberían tener los mismos derechos que las heterosexuales; sin embargo. menos del $30 \%$ está de acuerdo con que adopten hijos (Ministerio de Salud \& Profamilia. 2017). Estos resultados sugieren la existencia de prejuicios hacia personas LGB ( $p$. ej. pensar que no son suficientemente estables o responsables para la crianza) y a su vez nociones heteronormativas de las familias (Crawford. McLeod. Zamboni. \& Jordan. 1999; Pennington \& Knight. 2011). Por este motivo. es importante cuestionarse sobre las condiciones en las que habitan las familias homoparentales - aquellas encabezadas por padres o madres del mismo sexo-. y el tipo de obstáculos que encuentran en los contextos donde interactúan.

Se han realizado diversos estudios sobre actitudes hacia la homoparentalidad tanto con población general como con poblaciones específicas (p. ej. psicólogos/ trabajadores sociales/ docentes) (Costa y Salinas-Quiroz. 2018; Crawford et al.. 1999; Frias-Navarro. Monterde-i-Bort. Pascual-Soler. \& Badenes-Ribera. 2015; Weiner \& Zinner. 2015). Estos estudios revelan que ciertas características como el género. el grado de religiosidad. el tipo de tendencia política y las creencias relativas al origen de la homosexualidad. se han relacionado con estar más en contra de las familias homoparentales (Costa y Salinas-Quiroz. 2018; Frias-Navarro et al.. 2015). En países de América del Sur. como Colombia y Brasil. se conoce de esfuerzos investigativos sobre el tema (Campo. 2016; Castiblanco y Roa. 2019; Gusberti. Klaime. Kaufert. \& Silva. 2019). En conjunto. las investigaciones muestran un panorama fuertemente influido por la religión. creencias tradicionales sobre roles de género. así como una visión de la familia que privilegia la reproducción sexual y la estructura familiar de dos personas de distinto sexo (Lubbe. 2013).

Desde una perspectiva ecológica. uno de los contextos más importantes durante la niñez y la adolescencia de los hijos e hijas es el sistema educativo (Berns. 2013). En las instituciones educativas. niñas y niños fortalecen los aprendizajes recibidos en su hogar. aprenden contenidos dirigidos a su desarrollo cognitivo y se exponen a nuevas oportunidades de interacción con personas que contribuyen a la adquisición de habilidades. competencias. expectativas. valores y normas para la vida social (Berns. 2013; Vargas. 2013). Los años de preescolar. especialmente. son importantes por sus implicaciones en las trayectorias académicas. sociales y comportamentales de niñas y niños (Hamre \& Pianta. 2001).

A la fecha son escasos los estudios que analicen las actitudes hacia la homoparentalidad de docentes de preescolar (Church. Hegde. Averett. \& Ballard. 2016; Hegde. Averett. Parker White. \& Deese. 2014). El análisis de las actitudes de docentes en estos niveles es relevante por las implicaciones que

\footnotetext{
${ }^{1}$ La sigla LGBTIQ incluye a personas Trans (T), Intersexuales (I) y Queer (Q). Sin embargo, dado que el interés está en comprender las actitudes hacia la homoparentalidad, entendida a partir de la existencia de padres y madres con una orientación sexual lesbiana, gay o bisexual, en este artículo se utilizará la sigla LGB.
}

estas podrían tener a la hora de relacionarse con miembros de estas familias (Hedge et al.. 2014). En efecto. se ha encontrado que cuando los docentes no saben cómo trabajar con las familias o se sienten incómodos interactuando con ellas. madres y padres empiezan a sentirse menos importantes o excluidos de la comunidad educativa. Igualmente. cuando el personal docente no reflexiona sobre sus actitudes hacia la diversidad sexual y la homosexualidad. es más probable que adopten prácticas de silencio en el salón de clases que contribuyen a la invisibilidad. sobre todo en un momento cuando niñas y niños aprenden conceptos relevantes para su identidad como el de familia (Hegde et al.. 2014; Kintner-Duffy. Vardell. Lower. \& Cassidy. 2012).

Este estudio. por lo tanto. buscó describir a partir de una muestra de docentes de preescolar en Bogotá (Colombia) sus actitudes hacia las familias homoparentales. su percepción de autoeficacia para relacionarse y trabajar de forma inclusiva con ellas. así como las prácticas que adoptan o consideran útiles para favorecer la adaptación de niñas y niños al preescolar.

\section{Relaciones entre familias homoparentales y docentes}

Indiscutiblemente. las y los docentes juegan un papel importante durante los años de preescolar. El personal docente. por ejemplo. es responsable de construir ambientes de aprendizaje dirigidos a promover y potencializar el desarrollo y el bienestar de niñas y niños (Ministerio de Educación Nacional. 2014). Igualmente. son una pieza clave en la construcción de relaciones de cooperación de largo plazo con las familias. La evidencia revela que cuando existen relaciones caracterizadas por compromiso. comunicación y colaboración entre el contexto familiar y el contexto escolar. niñas y niños tienen mayores probabilidades de desarrollar un autoconcepto positivo. mejores habilidades sociales. emocionales y cognitivas; mejor desempeño académico y actitudes positivas hacia el trabajo escolar; comportamientos adaptativos; mayor participación en clases y mayor asistencia a la escuela (Hirst. Jervis. Visagie. Sojo. \& Cavanagh. 2011).

Lamentablemente. la evidencia demuestra que las y los docentes muchas veces no cuentan con las capacidades. las herramientas ni la formación para relacionarse de forma colaborativa e inclusiva con las familias homoparentales. Kosciw y Diaz (2008). por ejemplo. aplicaron una encuesta para indagar sobre las experiencias de 154 estudiantes entre 13 y 20 años. hijas e hijos de personas LGBT. Los resultados de este estudio revelaron que el personal docente puede propiciar situaciones que dificultan la integración y la inclusión de estudiantes que son miembros de estructuras familiares alternativas. así como su participación dentro de la vida escolar. En la muestra total se encontró que $30 \%$ de los estudiantes afirmaron sentir que no podían participar completamente en su escuela por la estructura de su familia. $22 \%$ habían recibido consejos y comentarios por parte de sus docentes para evitar hablar de sus familias y $36 \%$ sentía que sus maestros no reconocían sus familias como entornos familiares legítimos (por ejemplo. al no aceptar autorizaciones firmadas por la madre/padre no biológico). Igualmente. se encontró que las y los docentes pueden participar en situaciones de maltrato y discriminación más abiertas realizando en ocasiones comentarios derogatorios o prácticas excluyentes (e. g. no dejar a sus estudiantes realizar dos tarjetas para el día de la madre) (Kosciw \& Diaz. 2008). 
Asimismo. la evidencia indica que madres y padres también pueden ser víctimas de un trato inapropiado por parte del personal docente y otras familias. Investigaciones cualitativas han sugerido la existencia de prácticas docentes que desconocen abiertamente a las familias diversas entre las cuales se pueden citar el uso de materiales con sesgos heterosexistas. así como comportamientos inadecuados por parte de maestros y directivas. tales como miradas descalificadoras. no invitar a ambos padres o madres a eventos familiares y cuestionar las habilidades parentales (e. g. Lindsay et al.. 2006; Mercier \& Harold. 2003).

\section{Estudios sobre docentes de nivel preescolar}

Analizar las actitudes de docentes hacia la homosexualidad y la crianza por parejas del mismo sexo es fundamental puesto que sus posturas pueden contribuir a la invisibilidad y la exclusión de las familias. Efectivamente. cuando las y los docentes evitan hablar sobre este tipo de familias no solo transmiten ideas heteronormativas sobre la familia y la sexualidad. sino que pueden obstaculizar la comprensión de niñas y niños de su propia estructura familiar y transmitir un mensaje erróneo de que existe algo negativo con sus familias. lo que puede alimentar sentimientos de vergüenza. miedo o diferencia (Hegde et al.. 2014; Kintner-Duffy et al.. 2012).

Estudios en contextos norteamericanos indican que por lo general. el personal docente expresa actitudes positivas hacia la homosexualidad y la crianza en familias homoparentales (Averett \& Hegde. 2012; Averett et al.. 2015; Church. Hegde. Averett. \& Ballard. 2016). Se ha encontrado que las y los docentes suelen estar de acuerdo con que la crianza por personas homosexuales no afecta el bienestar de niñas y niños y que no debería existir discriminación hacia las personas basadas en su orientación sexual. Sin embargo. estas actitudes. al igual que en los estudios realizados con población general. varían de acuerdo con factores como el sexo. la edad. el nivel educativo y el grado de conservadurismo político. siendo las mujeres. las personas con niveles educativos altos. liberales y más jóvenes quienes expresan actitudes más positivas hacia la homoparentalidad (Averett \& Hegde. 2012).

Cabe mencionar que actitudes positivas no necesariamente significan comodidad ni confianza en el establecimiento de relaciones de trabajo con las familias. Averett y Hegde (2012). por ejemplo. encontraron que el personal docente no necesariamente se siente cómodo al trabajar con las familias. como consecuencia de la poca formación sobre estos temas durante los estudios de educación superior. la falta de recursos para informarse sobre sexualidad y homosexualidad. así como aspectos más personales como creencias religiosas y el desconocimiento sobre cómo hablar sobre diversidad sexual (p. ej. pensar que para hablar sobre orientación sexual es necesario hablar sobre actividad sexual penetrativa) (Kintner-Duffy et al.. 2012).

Estos resultados. en conjunto. sugieren la importancia que las y los docentes desarrollen un mayor sentido de autoeficacia para trabajar para y con personas sexualmente diversas y sus familias (Brant. 2014. 2017). La autoeficacia corresponde a la serie de creencias personales sobre las capacidades personales para ejecutar acciones requeridas para obtener un determinado resultado (Brant. 2017). En el caso de asuntos relativos a diversidad sexual. la autoeficacia implica la capacidad para relacionarse y trabajar de forma directa con estudiantes y fa- milias LGB. enseñar sobre temáticas relativas a estas poblaciones. así como la identificación y disminución de sesgos en el contexto educativo (Brant. 2014. 2017). Se ha encontrado que. si bien los docentes reportan sentir altos grados de autoeficacia. pueden sentir mayores dificultades para combatir los sesgos heterosexistas y planificar actividades que reduzcan prejuicios contra personas y familias LGB (Brant. 2017).

Otro hallazgo tiene relación con las dificultades de docentes para reconocer y abordar abiertamente los asuntos relacionados con diversidad familiar en el contexto de aula (Larrabee \& Kim. 2010). Esto es particularmente relevante durante el Preescolar. en la medida en que durante estos años. niñas y niños aprenden. comparten y hablan sobre su entorno familiar y su cotidianidad. desarrollan su modelo de "normalidad" y construyen su sentido de identidad y seguridad (Glass. Willox. Barrow. \& Jones. 2015). Los estudios al respecto revelan que las y los docentes tienden a desconocer e ignorar las múltiples configuraciones familiares de las cuales provienen sus estudiantes y asumen erróneamente que en sus familias existe un padre y una madre. Esto. sumado a su inseguridad y el uso permanente de recursos que ocultan la diversidad (por ejemplo. libros y películas que solo muestran familias heteroparentales). genera un contexto en donde niñas y niños no logran acceder a información relacionada con su propio entorno lo que puede transmitir el mensaje de que existen aspectos negativos en su núcleo familiar.

Finalmente. existen estudios en los que se discuten prácticas que pueden emplearse para generar espacios seguros para las familias homoparentales. Por ejemplo. se ha reportado que la realización de actividades y discusiones basadas en literatura en donde se muestra explícitamente distintas formas de familia puede generar conversaciones entre docentes y estudiantes que contribuyen a neutralizar las normas heterosexistas del contexto, reconocer las múltiples formas en que se manifiesta la diversidad humana y la amplitud de formas que pueden caracterizar a las relaciones y los vínculos entre seres humanos (por ejemplo. la adopción) (Souto-Manning \& Hermann-Wilmarth. 2008). Este tipo de actividades beneficia a niñas y niños puesto que genera un espacio a través del cual maestras y maestros pueden ingresar a su mundo cotidiano. valorarlo y reconocerlo como un aspecto fundamental de su identidad. Igualmente. es a través de este tipo de actividades que las instituciones educativas pueden contribuir a generar espacios seguros entre niñas y niños. en los cuales se visibilice la diversidad y se enseñe el respeto por las diferencias. transmitiéndoles un mensaje de bienvenida y aceptación a todo tipo de personas y configuraciones familiares.

\section{Homoparentalidad en Colombia}

En Colombia se han realizado muy pocos estudios sobre las experiencias de familias homoparentales. En el campo de la Psicología cabe destacar el trabajo realizado por Vargas TrujiIlo. Ripoll. Carrillo. Rueda Sáenz y Castro (2011). en el cual analizaron las experiencias de madres y padres no heterosexuales con relación a sus familias de origen y sus hijos. En la literatura revisada para esta investigación. no se encontraron estudios sobre las experiencias de familias colombianas en contextos como escuelas y colegios.

Realizar más estudios sobre las experiencias de familias homoparentales en el país es imperativo considerando la serie de cambios que se han dado frente a la conformación y el re- 
conocimiento legal de estas formas de familia. En 2015. por ejemplo. la Corte Constitucional aprobó la eliminación de los obstáculos jurídicos para la solicitud de adopciones por parte de parejas del mismo sexo. Si bien no existen estadísticas oficiales del número de adopciones solicitadas y concretadas. es posible que progresivamente se incremente el número de familias homoparentales en el país y que incremente la visibilidad de familias ya existentes como consecuencia de la percepción de mayor seguridad y reconocimiento jurídico.

Por otro lado. con relación al ámbito educativo. la muerte de un estudiante de secundaria en 2014 se constituyó en un caso emblemático que trajo cuestionamientos sobre qué tan seguras son las instituciones educativas para personas sexualmente diversas (Herrera Durán. 2014). A partir de este caso. la Corte Constitucional promulgó la Sentencia T478/15 a través de la cual se ordenó a los planteles educativos del país revisar sus manuales de convivencia para prohibir la discriminación basada en la orientación sexual o la identidad de género de sus estudiantes (Corte Constitucional de Colombia. 2015). En respuesta a estas medidas. se llevaron en el país protestas en defensa de la familia tradicional y en contra de la llamada 'ideología de género' (Cullinan Hoffman. 2016). Estos acontecimientos. por lo tanto. revelan la polarización del entorno colombiano y lo importante que se torna contar con evidencia que permita visibilizar las experiencias de familias homoparentales. A partir de la realización de más estudios. será posible enriquecer los debates relativos a estas formas de familia y contribuir a la toma de decisiones para la construcción de entornos educativos seguros.

En este contexto. este estudio buscó responder las siguientes preguntas de investigación: ¿Qué actitudes tienen docentes de Preescolar hacia la crianza de niñas y niños por parte de personas homosexuales? ¿Cómo se perciben los docentes en relación con su autoeficacia para trabajar con familias homoparentales? ¿Qué relaciones existen entre las actitudes hacia la homoparentalidad y los niveles de autoeficacia de docentes para el trabajo con estas familias? y ¿Qué prácticas usan o consideran que podrían usar los docentes con relación al trabajo con las familias homoparentales?

\section{Método}

\section{Diseño}

Este estudio utilizó un diseño transversal descriptivo.

\section{Participantes}

La muestra fue por conveniencia y se utilizaron dos estrategias de muestreo. En primer lugar. se solicitó la colaboración de la Facultad de Educación de una universidad privada en Bogotá para diseminar una invitación a sus estudiantes de posgrado que cumplieran con los criterios de inclusión. Estos criterios eran: 1) ser docente de nivel preescolar al momento de participar. es decir. en Pre-jardín (3 años). Jardín (4 años) y Grado Cero (5 años). y 2) encontrarse trabajando como docente en cualquier institución pública o privada de la ciudad de Bogotá. La segunda estrategia consistió en contactar a conocidos de la red personal del autor que mantenían contacto con instituciones educativas.

La muestra final estuvo conformada por 50 mujeres (94.3\%) y 3 hombres (5.7\%). La edad promedio de las personas fue de 38.3 años (D. E. = 10.85). La mayoría reportó ser hetero- sexual (98.1\%) y al momento de ser encuestadas reportaron vivir y trabajar en la ciudad de Bogotá (100\%). En cuanto a su nivel educativo. 30 personas reportaron tener estudios universitarios completos (53.6\%). 13 (23.2\%) manifestaron tener una especialización concluida y solamente 5 reportaron tener estudios de maestría terminados (8.9\%). La mayoría (39.62\%) reportó que su campo de formación principal fue el área de educación. específicamente en aspectos de desarrollo infantil y pedagogía a nivel preescolar.

En cuanto a su actividad laboral. en promedio. las personas contaban con 14.2 años $(D . E .=10.7)$ de experiencia docente y al momento de responder la encuesta. la mayoría trabajaba solamente en una institución educativa (83\%). Las localidades donde laboraba la mayoría fueron Suba (24.5\%) y Usaquén (22.6\%). En cuanto al tipo de institución educativa. la mayoría reportó estar trabajando en instituciones privadas (83\%) y laicas (67.9\%). El promedio de años de afiliación a la institución educativa actual fue de 6.97 años $(D . E .=6.7)$.

\section{Instrumentos}

Escala de Creencias sobre el Ajuste de Niños en Familias Homoparentales (Frías-Navarro. 2009). La versión empleada contó con 13 ítems y mide. a través de una escala tipo Likert de 1 (totalmente en desacuerdo) a 5 puntos (totalmente de acuerdo). las creencias sobre los efectos de la crianza por parte de personas gay y lesbianas en niñas y niños. El puntaje mínimo es de 13 puntos y el máximo de 65 puntos.

La escala permite obtener dos puntajes: oposición individual. la cual mide el grado de acuerdo a ideas personales que manifiestan desagrado o una posición negativa hacia la posibilidad de que parejas del mismo sexo desempeñen tareas de crianza; y oposición normativa. la cual "operacionaliza situaciones que los ciudadanos han internalizado como 'normales'. 'naturales' o 'aceptables'. no siendo conscientes de su propio prejuicio" (Frías-Navarro. 2009. p. 2). De esta manera. el instrumento permite obtener tres puntajes distintos: uno global y dos específicos (oposición individual y normativa). Análisis psicométricos con la muestra del estudio reflejan altos puntajes de confiabilidad para la escala global $(\alpha=.928)$ y para las escalas de oposición individual ( $\alpha=.893$ ) y oposición normativa $(\alpha=.891)$. Estos puntajes son similares a los obtenidos en estudios previos en los cuales se han encontrado alfas que oscilan entre 0.88 y 0.92 para las subdimensiones que evalúa el instrumento (Frias Navarro et al.. 2015).

Escala de Autoeficacia Docente en Temas LGBTIQ (Brant. 2014). Esta escala fue desarrollada por Brant (2014) para medir la percepción de autoeficacia para el trabajo con familias homoparentales. Esta escala mide a partir de 8 ítems Likert (1 = nada seguro/a; 4 = completamente seguro/a). las creencias que poseen los docentes sobre su capacidad de organizar y ejecutar acciones de docencia con particular énfasis en el trabajo con estudiantes y familias LGBTIQ. la enseñanza sobre temáticas LGBTIQ y la identificación de sesgos en los materiales usados en el aula y en general. el contexto educativo (Brant. 2017). El puntaje puede ser entre 8 puntos y 32 puntos. Datos psicométricos con la muestra de este estudio demuestran una confiabilidad alta $(\alpha=0.904)$.

Cuestionario de Prácticas Docentes. Este cuestionario fue desarrollado para este estudio y evaluó el grado en que el personal docente lleva a cabo acciones para favorecer la adap- 
tación de niñas y niños al preescolar y el trabajo conjunto con familias. Específicamente. se evaluó qué tan posible podría ser el llevarlas a cabo. qué tan útiles consideran que pueden ser y qué tan seguros/as se sienten para llevarlas a cabo. La lista de prácticas incluyó 5 ítems que incluían: el desarrollo de encuentros con padres y madres (incluidas parejas del mismo sexo). la organización del día de la familia incluyente. la realización de jornadas para entrega de notas en donde se invite a ambos miembros de parejas del mismo sexo. entre otras.

Igualmente. con el fin de indagar por estrategias relacionadas con el tema de la familia. se solicitó a las personas identificar distintas formas de familia (e. g. hombre soltero sin hijos. pareja de hombre y mujer con mascota. pareja de mujer y mujer con hijo) y reportar si son ejemplos que emplean en el aula. La lista fue adaptada a partir de los ejemplos utilizados por Larrabee \& Kim (2010).

\section{Procedimientos}

La encuesta fue administrada por medio de dos vías: virtual y física. Algunas personas fueron contactadas por medio de correo electrónico y recibieron un vínculo a la encuesta. Para acceder al cuestionario. las personas debían leer y aceptar el formulario de consentimiento informado. Las personas que respondieron a la versión física de la encuesta lo hicieron en espacios asignados por cada institución educativa. Análisis posteriores demostraron que los resultados no difieren estadísticamente al compararlos según el medio de administración (virtual vs. físico). Todas las aplicaciones fueron en horas de la mañana en sesiones de 45-60 minutos. Una vez finalizada la aplicación física. se destinaron entre 15 y 20 minutos para conocer las reacciones frente al tema del estudio. los contenidos del cuestionario y las emociones experimentadas por las personas participantes durante la encuesta.

\section{Análisis de datos}

Para responder a las preguntas de investigación se realizaron análisis descriptivos. correlacionales. así como pruebas t de muestras independientes. Para comparar las correlaciones se consideró los siguientes valores q de Cohen: menor a 0.10 no hay efecto; entre 0.10 a 0.30 . efecto pequeño; 0.31 a 0.50 . efecto moderado y mayor 0.51 efecto grande (Ventura-León \& Caycho. 2017).

\section{Resultados}

P1: ¿Qué actitudes tienen docentes de preescolar hacia la crianza de niñas y niños por parte de personas homosexuales?

Las personas participantes expresaron actitudes neutras hacia la crianza en familias homoparentales $(M=35.52 ; D . E$. $=$ 12.94). Al comparar los índices de oposición normativa $(M=$ $20.04 ; D . E .=6.97)$ con el de oposición individual $(M=15.67$; $D$. $E$. $=7.44)$ se encontró una diferencia estadísticamente significativa (t $(48)=-6.18 ; p=.00$ ) lo cual indica que las y los docentes tendieron a estar más de acuerdo con creencias comunes en el entorno sociocultural sobre las familias homoparentales. que afirmaciones que demuestran ideas personales de desagrado y rechazo hacia la existencia de estas familias. Como muestra la figura 1. el análisis de frecuencias demostró que las afirmaciones con mayor grado de acuerdo son aquellas que hacen referencia a la probabilidad de que niñas y niños. por tener padres y madres homosexuales. posean mayor riesgo de aislamiento. exclusión y victimización (por ejemplo. burlas) por parte de sus pares. Otras afirmaciones con altos grados de acuerdo revelan la creencia de que niñas y niños pueden sentir la necesidad de ocultar la orientación sexual de sus madres/padres (y por ende su estructura familiar) a fin de evitar repercusiones negativas. o la importancia de que niñas y niños visiten un/a psicólogo/a en caso de que existan indicios de una orientación no heterosexual.

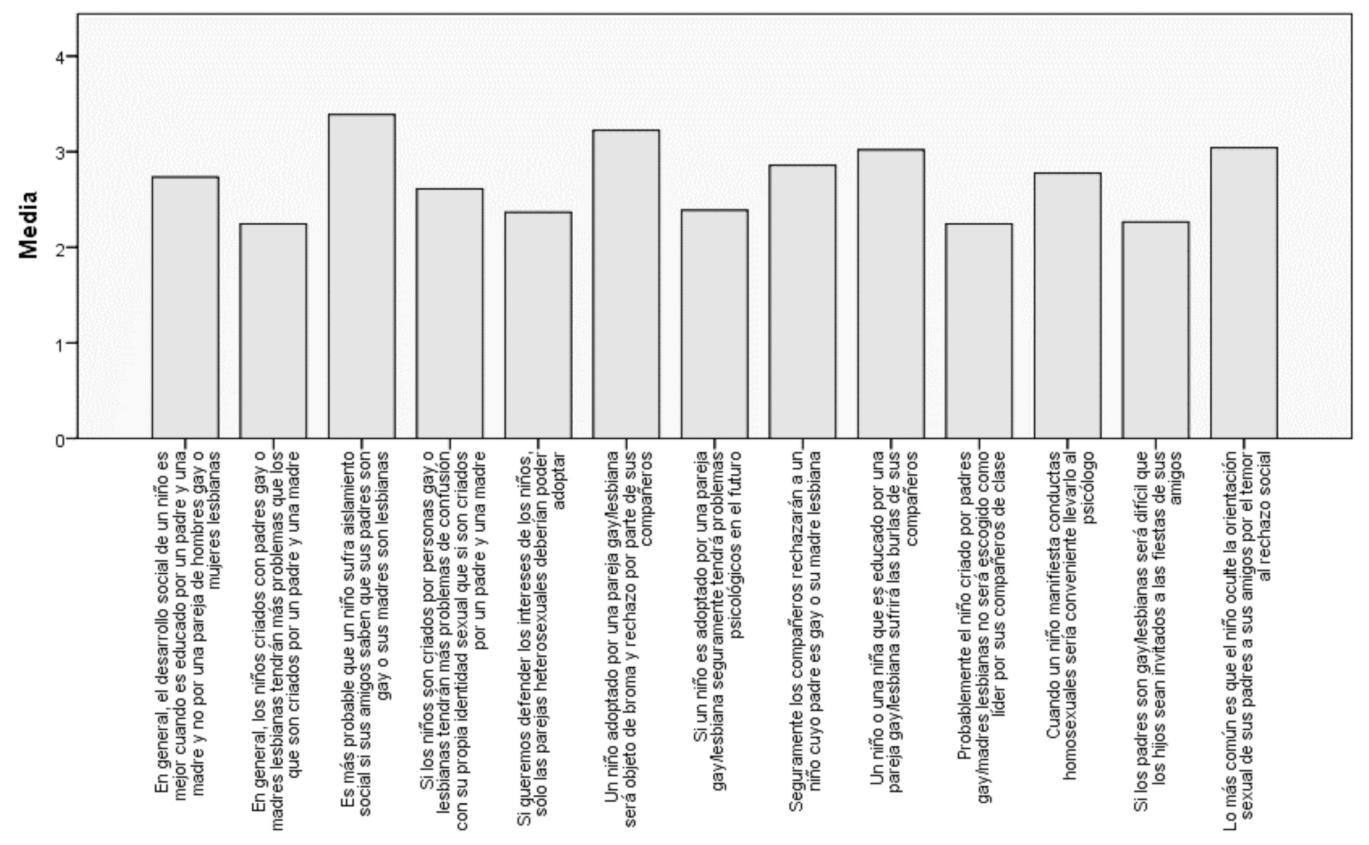

Figura 1. Actitudes hacia la crianza de hijas e hijos en familias homoparentales 
P2: ¿Cómo se perciben los docentes en relación con su autoeficacia para trabajar conjuntamente con las familias homoparentales?

Con relación a las percepciones de autoeficacia de las y los docentes. se encontró una media de 27.44 puntos (D.E. =
5.19). Como lo muestra la figura 2. la mayoría de las personas (23.1\%) obtuvieron el puntaje máximo de la escala.

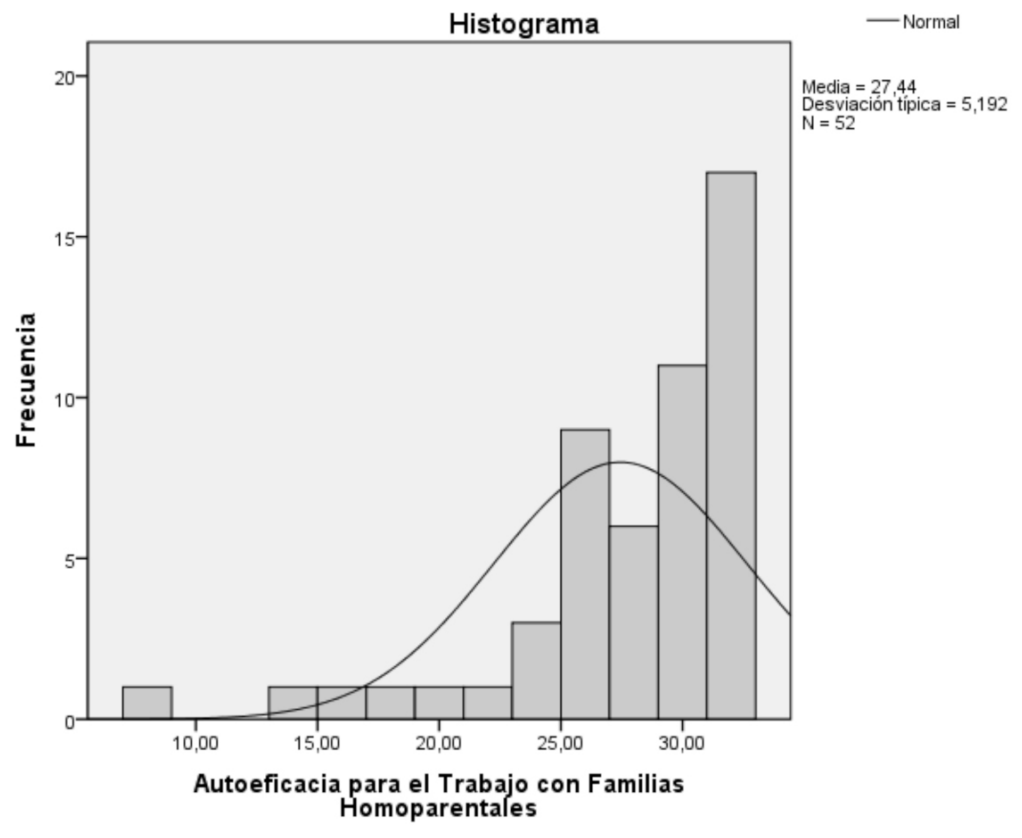

Figura 2. Distribución de medias de la percepción de autoeficacia para el trabajo con familias homoparentales

Como indica la tabla 1. los aspectos en los que se sienten más seguras las personas se refieren a identificar e informar situaciones de maltrato hacia niñas y niños. Efectivamente. $94.2 \%$ de las personas encuestadas reportaron sentirse seguras frente a este aspecto. Los aspectos en los cuales se sienten más inseguras tienen relación con identificar prácticas y políticas institucionales que afectan a personas gais y lesbianas (13.4\%). pero principalmente ayudar a generar cambios de esas políticas (19.2\%).

Tabla 1. Percepción de autoeficacia para el trabajo con familias homoparentales

\begin{tabular}{|c|c|c|}
\hline & $f$ & $\%$ \\
\hline $\begin{array}{l}\text { Informar a autoridades de la institución educativa situaciones de maltrato físico o psicológico por cuestiones } \\
\text { de la orientación sexual de padres/madres }\end{array}$ & 50 & 96.15 \\
\hline Identificar situaciones de maltrato físico o psicológico contra los hijos de personas gais o lesbianas & 49 & 94.23 \\
\hline Planificar actividades de docencia dirigidas a reducir el prejuicio contra personas gais y lesbianas & 48 & 92.31 \\
\hline $\begin{array}{l}\text { Informar a padres/madres situaciones de maltrato físico o psicológico por cuestiones de la orientación sexual } \\
\text { de padres/madres }\end{array}$ & 47 & 90.38 \\
\hline Implementar actividades de docencia dirigidas a reducir el prejuicio contra personas gais y lesbianas & 46 & 88.46 \\
\hline $\begin{array}{l}\text { Identificar sesgos y contenidos estereotipados sobre las personas gais y lesbianas en materiales de clases ( } p \text {. } \\
\text { ej. libros) }\end{array}$ & 44 & 84.62 \\
\hline Identificar prácticas y políticas en la institución educativa que afectan el bienestar de personas gais y lesbianas & 43 & 82.69 \\
\hline $\begin{array}{l}\text { Ayudar a cambiar prácticas y políticas de institución educativa que afectan el bienestar de personas gais y } \\
\text { lesbianas }\end{array}$ & 40 & 76.92 \\
\hline
\end{tabular}

Nota: *La tabla muestra el número de personas que reportaron sentirse seguras o muy seguras 
P3: ¿Qué relaciones existen entre las actitudes hacia la homoparentalidad y los niveles de autoeficacia de docentes para el trabajo con estas familias?

El análisis de correlaciones reveló la existencia de relaciones negativas entre los puntajes de la escala de actitudes hacia la homoparentalidad con los puntajes de autoeficacia percibida de docentes $(r=-0.284 ; p<0.05)$. Se encontró que cuando las personas expresan actitudes más negativas. se perciben menos capaces para trabajar asuntos que permiten construir ambientes seguros para la diversidad sexual. El cálculo de la q de Cohen (-0.127) indicó un efecto pequeño de la diferencia entre las correlaciones de las escalas de oposición individual $(r=-0.375 ; p<0.01)$ y oposición normativa $(r=-0.261 ; p>$ $0.05)$; el intervalo de confianza de estas puntuaciones. sin embargo. indica que la diferencia no fue significativa (ICSup = $0.10 ; \mathrm{IC} \operatorname{lnf}=-0.36$ ).
P4: ¿Qué prácticas usan o consideran que podrían usar los docentes con relación al trabajo con las familias homoparentales?

Por último. se indagó por el tipo de prácticas que las y los docentes consideran que podrían emplear en sus instituciones educativas. el grado de utilidad de esas prácticas. así como su nivel de confianza para llevarlas a cabo. Como lo indica la tabla 2. los resultados muestran que aproximadamente el $80 \%$ de las personas sienten que es posible y útil realizar encuentros con todos los padres y madres. incluidas las parejas del mismo sexo. antes de empezar el año escolar. realizar jornadas de entrega de notas. facilitar el contacto entre familias. así como invitarlas a participar en las actividades pedagógicas que se llevan a cabo en el aula. Igualmente. la mayoría de docentes se sienten seguras/os de poder llevar a cabo estas tareas.

Tabla 2. Evaluación de posibilidad de ejecución. utilidad percibida y autoeficacia para llevar a cabo prácticas en el trabajo con familias homoparentales

\begin{tabular}{|c|c|c|c|c|c|c|}
\hline & \multicolumn{2}{|c|}{$\begin{array}{c}\text { Muy } \\
\text { probable }\end{array}$} & \multicolumn{2}{|c|}{ Muy útil } & \multicolumn{2}{|c|}{ Muy seguro/a } \\
\hline & $f$ & $\%$ & $f$ & $\%$ & $f$ & $\%$ \\
\hline $\begin{array}{l}\text { Un encuentro con todos los padres y ma- } \\
\text { dres del salón. incluidas parejas del mismo } \\
\text { sexo (ambos miembros de la pareja). antes } \\
\text { de empezar el año escolar }\end{array}$ & 33 & 70.2 & 34 & 73.9 & 32 & 68.1 \\
\hline $\begin{array}{l}\text { Realizar las jornadas de entregas de notas } \\
\text { a las que asistan parejas del mismo sexo } \\
\text { (ambos miembros de la pareja) }\end{array}$ & 33 & 70.2 & 38 & 80.9 & 35 & 72.9 \\
\hline $\begin{array}{l}\text { Facilitar el contacto entre los padres y ma- } \\
\text { dres de familia de los estudiantes. inclui- } \\
\text { das parejas del mismo sexo (ambos miem- } \\
\text { bros de la pareja) }\end{array}$ & 32 & 68.1 & 35 & 76.1 & 30 & 62.5 \\
\hline $\begin{array}{l}\text { Invitar a los padres de familia. incluidas pa- } \\
\text { rejas del mismo sexo. a hacer parte de las } \\
\text { actividades pedagógicas durante la jorna- } \\
\text { da escolar }\end{array}$ & 32 & 68.1 & 37 & 80.4 & 34 & 72.3 \\
\hline $\begin{array}{l}\text { Organizar el día de la familia. en el que se } \\
\text { incluyan las familias de padres/madres }\end{array}$ & 32 & 68.1 & 37 & 80.4 & NA & NA \\
\hline
\end{tabular}

Nota: *La tabla muestra el número de personas que afirmaron que era "muy probable". "muy útil" ejecutar la práctica. así como sentirse "muy seguro/a" para llevarla a cabo.

Adicionalmente. con el fin de identificar si las y los docentes realizan prácticas dirigidas a reconocer la diversidad de las familias en sus aulas. se les solicitó identificar distintos tipos de familia y reportar si usan esos ejemplos en clase. El ejemplo más utilizado en clase es el de la familia tradicional de madre. padre e hijos. Aproximadamente $80 \%$ de las personas reconocieron los ejemplos de parejas de dos hombres y dos mujeres con hijos como una forma de familia. Sin embargo. como 


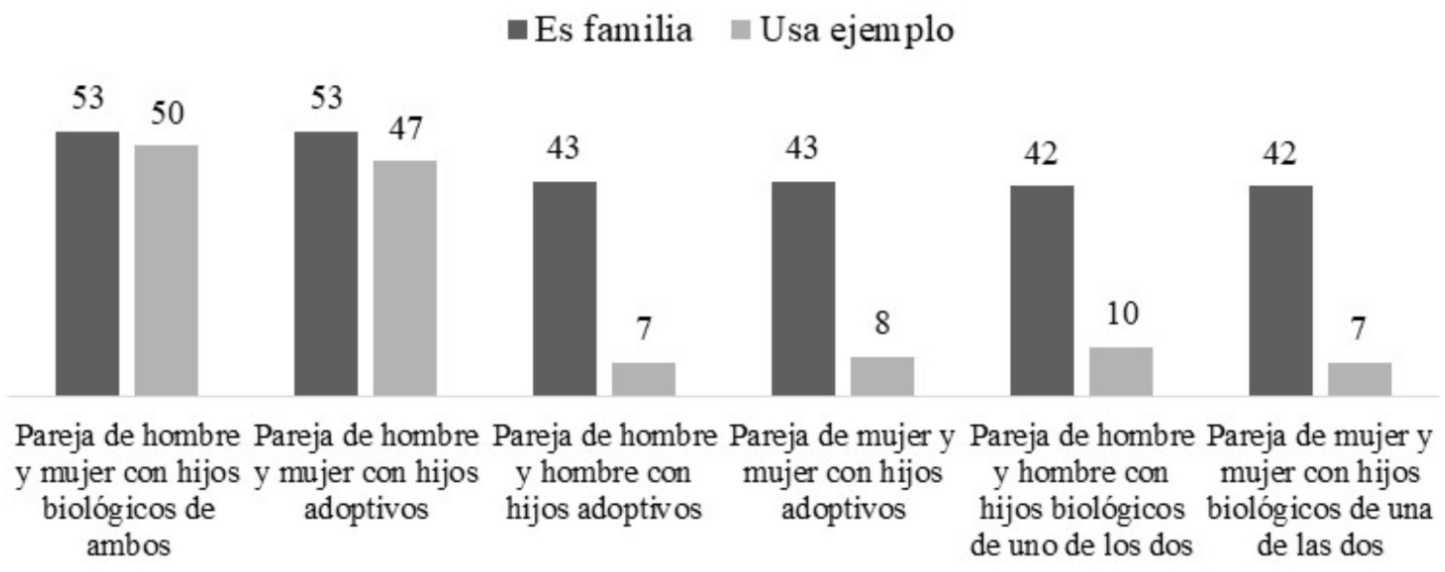

Figura 3. Reconocimiento de formas de familia y empleo de ejemplos en clase $(n=53)$

\section{Discusión}

Este estudio tuvo como objetivo identificar las actitudes hacia familias homoparentales de docentes de preescolar. sus percepciones de autoeficacia relativas al trabajo con estas formas de familia al igual que las prácticas que utilizan o consideran que podrían usar a la hora de trabajar con ellas. Para cumplir con este propósito se aplicó una encuesta a docentes de pre-jardín. jardín y grado cero de instituciones públicas y privadas de Bogotá.

El análisis de las actitudes de las y los docentes es fundamental por sus relaciones con la construcción de climas escolares inclusivos y libres de discriminación. Kintner-Duffy. Vardell. Lower y Cassidy (2012) indican que "la falta de educación. los mitos. las creencias morales/religiosas y el miedo [...] crean una resistencia que debe ser atendida con el fin de que los programas educativos puedan cumplir con sus estándares éticos de respeto por el niño y su familia" (p. 212). En el contexto colombiano esto es relevante considerando los principios que sustentan la educación inicial del país en términos del reconocimiento de las familias como figuras que aportan saberes y prácticas a la tarea educativa y las y los docentes como figuras que configuran entornos que potencializan el desarrollo de niñas y niños (Ministerio de Educación Nacional. 2014). Por esta razón se considera que solo a través de la reflexión. la adquisición de información y el cuestionamiento de los mensajes socioculturales relativos a la diversidad sexual y familiar es posible que desde las instituciones educativas se implementen prácticas que garanticen el derecho a una educación libre de prejuicios. que fomente la participación y el desarrollo de un trabajo conjunto entre familias y escuelas.

En cuanto a las actitudes hacia la homoparentalidad se encontró que las y los docentes mostraron actitudes neutras. Es posible que estos resultados reflejen una posición imparcial frente a la crianza por parejas del mismo sexo. pero también pueden expresar: a) una actitud ambivalente o indecisa; b) el uso del puntaje intermedio de la escala como una manera de evadir la respuesta; c) una forma de evitar comprometerse con una posición o una opinión definida a favor o en contra; o d) una manera de responder con cautela frente a un tema claramente sensible.

Un análisis más detallado de los resultados permitió diferenciar las actitudes de acuerdo con las dos subescalas propuestas por Frías-Navarro (2009). esto es. la escala de oposición normativa y oposición individual. Al analizar estos puntajes. se encontró una diferencia estadísticamente significativa entre las escalas que indicaría que las personas participantes del estudio. en promedio. estuvieron más de acuerdo con expresiones que reflejan preocupaciones sobre los efectos para niñas y niños de ser criados por madres o padres LGB. Específicamente. se encontró que las y los docentes expresaron mayor acuerdo con creencias relativas a la mayor probabilidad de exclusión. aislamiento y discriminación a la que podrían enfrentarse niñas y niños.

Estudios previos sobre actitudes han revelado resultados similares en la medida que los participantes reportan frecuentemente preocupación por fenómenos como el matoneo por homofobia (Pennington \& Knight. 2011). De hecho. este es uno de los argumentos más utilizados en las discusiones sobre la adopción por parejas del mismo sexo y transmite la idea de que el ejercicio de la paternidad/maternidad debería ser negado a personas homosexuales con el fin de evitar posibles consecuencias negativas en niñas y niños (Pennington \& Knight. 2011). Este argumento parecería que en últimas funciona como un heurístico a través del cual se generan conclusiones sobre las familias homoparentales (e. g. 'no debería haber padres homosexuales para evitar el matoneo') evitando que las personas cuestionen el verdadero origen de los problemas a los que pueden estar sujetos los miembros de estas familias. esto es. las creencias culturales alrededor de la homosexualidad y la homoparentalidad. Por este motivo. el no cuestionamiento de este tipo de creencias trae como consecuencia la perpetuación de estereotipos que se encuentran implícitos (i. e. 'los hogares heteroparentales son mejores') y que contribuyen en últimas a generar un estigma hacia los miembros de estas familias. Sería deseable. por lo tanto. que futuros estu- 
dios analicen con mayor profundidad las razones por las cuales docentes expresan este tipo de preocupaciones.

Adicionalmente. el punto anterior permite reflexionar sobre la manera en que las y los docentes se perciben a sí mismas/os en relación con su rol como agentes responsables de promover el bienestar de niñas y niños. En otras palabras. si efectivamente las personas expresan preocupación sobre lo que puede ocurrir con menores criados en familias homoparentales. ¿cómo se perciben en relación con la creación de ambientes seguros para esos niñas y niños? ¿qué pueden hacer con el fin de evitar que ocurran situaciones de discriminación. exclusión y asilamiento?

Los resultados del estudio sugieren que las y los docentes. por lo general. se sienten seguros de su capacidad para trabajar con familias homoparentales. y específicamente. para identificar y reportar situaciones de maltrato por homofobia dirigido a hijas e hijos de estas familias. Este resultado. si bien es positivo en la medida que revela autoeficacia para construir climas escolares seguros. debe ser analizado con cautela. Efectivamente. existe evidencia que demuestra que la victimización hacia niñas y niños de familias homoparentales tiende a ser baja en preescolar y que el personal docente puede llegar a percibir que este no es un problema importante para resolver durante estos años de la educación formal (Autor. 2017). Por esta razón. si las y los docentes consideran que este fenómeno es inexistente. es posible que en últimas se desaproveche la oportunidad de enseñar a niñas y niños sobre las múltiples formas que adopta la diversidad. así como el respeto y la aceptación de las diferencias entre personas. Como mencionan Glass y colegas (2015) se ha encontrado que "los niños de tan solo dos años son capaces de reconocer las diferencias en su entorno social y que existen sentimientos positivos o negativos hacia estas diferencias" (p. 220). Por este motivo. es fundamental que el personal docente de preescolar sea capaz de reconocer que trabaja con niñas y niños de edades en las cuales es posible generar un impacto positivo a través de la discusión y cuestionamiento de los mensajes que se transmiten a nivel sociocultural (Glass et al.. 2015; Souto-Manning \& Hermann-Wilmarth. 2008).

Una de las estrategias que se ha identificado para promover la construcción de entornos seguros es la presentación y la reflexión en el aula sobre las distintas formas de familia (Souto-Manning \& Hermann-Wilmarth. 2008; White. 2015). Los resultados del estudio muestran que. si bien $80 \%$ de las personas participantes reconocieron a las familias homoparentales como una forma de familia. solamente entre el 5 y $18 \%$ reportó utilizar este tipo de familia como ejemplo para abordar el concepto de familia en el aula. La gran disparidad entre el reconocimiento y el uso de ejemplos sobre familias homoparentales es preocupante. pudiendo reflejar falta de disposición o preparación de las y los docentes para realizar discusiones alrededor de estas formas de familia. Del mismo modo. puede indicar temores que el personal docente puede sentir. tales como exponerse a críticas o quejas de parte de directivas $u$ otros miembros de la comunidad educativa como madres y padres de familia. Se han reportado miedos como ser despedidos o reprendidos por trabajar sobre temas de diversidad sexual. ser llamados homosexuales o 'gay-friendly' así como cuestionados en términos morales o religiosos (White. 2015). Por esta razón. sería recomendable continuar analizando por qué las y los docentes no emplean ejemplos sobre este tipo de familias en el aula a fin de generar entornos en donde todos los estudiantes puedan recibir información sobre su estructura familiar y en donde puedan compartir. sin temor. datos sobre el núcleo del cual provienen. Sería provechoso que estas investigaciones profundicen. por ejemplo. en las características de las comunidades educativas y otros factores a fin de comprender las decisiones de las y los docentes sobre qué y cómo hablar de la familia en el aula.

Finalmente. el análisis de las escalas de autoeficacia reveló que las y los docentes expresaron altos niveles de seguridad para trabajar con familias homoparentales. sobre todo en lo relativo a la discusión de aspectos académicos y del desarrollo de niñas y niños. Es probable que esto se deba a que las personas perciben que. en últimas. son aspectos que tienen como centro al estudiante y que no varían según la orientación sexual de sus figuras parentales. Los aspectos en donde se reportaron menores puntajes de seguridad fueron aquellos referentes a la discusión de aspectos propios de la vida de cada familia como. por ejemplo: las creencias religiosas. la historia de conformación familiar y el estatus de la unión entre las figuras parentales. Si bien no es claro si esta es una dificultad generalizada o específica por tratarse de familias homoparentales. es probable que las y los docentes consideren que en su práctica cotidiana sea innecesario profundizar sobre estos aspectos posiblemente por creencias relacionadas con los límites de su labor profesional y no querer indagar sobre temáticas que pueden ser percibidas como privadas.

Frente a esto. analizar a mayor profundidad la serie de creencias detrás de estos resultados puede ser importante a fin de obtener más pistas del por qué estos tópicos se consideran particularmente difíciles o sensibles de abordar. Es posible que algunas personas consideren erróneamente que dentro de las familias homoparentales no se inculcan creencias religiosas o que las figuras parentales. por no estar civilmente unidas o casadas. no se identifiquen a sí mismas como una pareja formalmente constituida. Conocer estos aspectos de la vida familiar. y sentirse cómodos/as a la hora de indagar por la vida de las familias. puede ser una estrategia que en últimas genere mayor conocimiento sobre el entorno que rodea al niño y permita adaptar el trabajo de forma tal que se tomen en cuenta esas características individuales. Consistente con el enfoque de derechos y los lineamientos en los cuales está basada la educación preescolar en el país (Ministerio de Educación Nacional. 2014). analizar el contexto en el que viven niñas y niños es una tarea fundamental con el fin de tomar decisiones curriculares que reconozcan y se adapten a sus particularidades.

A partir de lo anterior. una estrategia para la construcción de entornos seguros es justamente el análisis de las creencias y actitudes de los docentes frente a la homoparentalidad y la diversidad sexual. En el caso de este estudio. se encontraron relaciones entre los puntajes de actitudes y los niveles de autoeficacia. Por lo tanto. se considera que el desarrollo de iniciativas que permitan a los docentes acceder a información y además oportunidades de cuestionamiento y análisis de actitudes personales. puede ser una manera efectiva de incrementar sus competencias para interactuar y colaborar con estas formas de familia.

Desde luego. este estudio cuenta con limitaciones. Una muy importante es que los docentes fueron en su mayoría de insti- 
tuciones privadas. seleccionadas por conveniencia. Esto hace que las conclusiones deban ser interpretadas con cautela. ya que es posible que una muestra mayor en la cual estén representados más docentes de instituciones públicas y de diversas zonas de Bogotá permita llegar a conclusiones distintas. Otra limitación se relaciona con los cuestionarios empleados. Debido a la escasez de cuestionarios sobre el tema. se recopilaron instrumentos desarrollados en otros contextos. Si bien se hizo un pilotaje y ajustes a los instrumentos. es recomendable que futuras investigaciones dediquen mayor atención a analizar la pertinencia de las escalas e ítems. Una forma de hacerlo. por ejemplo. es a través de entrevistas con docentes que permitan recopilar información de sus perspectivas sobre las experiencias en preescolar en niños de familias no tradicionales. Este tipo de procedimientos facilitaría complementar los instrumentos con ítems sensibles al contexto y a la población de estudio.

Por otra parte. sería deseable que futuras investigaciones indaguen de manera cualitativa sobre las creencias. actitudes. percepciones y prácticas de docentes. Los datos recopilados sugieren que existen múltiples opiniones que no necesariamente se reflejan a través de las encuestas. Por ejemplo. durante una de las aplicaciones. una de las participantes decidió escribir las razones por las cuales. en su opinión. las personas homosexuales no deberían criar niñas y niños (por ejemplo. 'La homosexualidad es algo que va en contra del plan de Dios'). Este tipo de situaciones demuestra la importancia de seguir profundizando sobre lo que piensan los distintos actores que interactúan durante la transición de niñas y niños.

En conclusión. es necesario continuar indagando por la serie de actitudes. percepciones y prácticas que tienen docentes con respecto a formas alternativas de familia. Las familias homoparentales son cada vez más visibles dentro de distintos contextos y ciertamente. el educativo. es uno de ellos. Por este motivo. si el objetivo es ofrecer a niñas. niños y familias un ambiente seguro e inclusivo. es necesario comenzar por identificar lo que el personal de las instituciones educativas piensa frente a estos temas y cómo. desde las prácticas dentro y fuera del aula. es posible contribuir a que las familias sean bienvenidas. incluidas y aceptadas. Esto. ciertamente. puede beneficiar a niñas y niños.

\section{Referencias}

Averett. P. \& Hegde. A. (2012). School social work and early childhood student's attitudes toward gay and lesbian families. Teaching in Higher Education. 17(5). 537-549.

Averett. P.. Hegde. A. \& Smith. J. (2015). Lesbian and gay parents in early childhood settings: A systematic review of the existing research literature. Journal of Early Childhood Research. 1476718X15570959.

Berns. R. (2013). Ecology of the child. In Child. Family. School. Community Socialization and Support (9th ed.. pp. 3-31). Wadsworth: Cengage Brain.

Brant. C. (2014). Pre-service teachers' perpectives on methods. pedagogy and self-efficacy related to gender and sexuality as part of their multicultural teacher education. The Ohio State University.

Brant. C. (2017). How Do I Understand the Term Queer? Preservice Teachers. LGBTQ Knowledge. and LGBTQ Self-Efficacy. Educational Forum. 81(1). 35-51.
Campo. A. (2016). Aceptación de la adopción por hombres homosexuales en estudiantes de medicina. Revista Colombiana de Enfermería. 6(6). 29.

Castiblanco. A. \& Roa. M. (2019). Actitudes hacia la adopción homoparental: Un estudio comparativo entre padres de familia de una institución educativa y jóvenes universitarios. Universidad Santo Tomás.

Church. J.. Hegde. A.. Averett. P. \& Ballard. S. (2016). Early childhood administrators' attitudes and experiences in working with gay- and lesbian-parented families. Early Child Development and Care. 1-17.

Corte Constitucional de Colombia. (2015). Sentencia T-478-15.

Costa. P. \& Salinas-Quiroz. F. (2018). A Comparative Study of Attitudes Toward Same-Gender Parenting and Gay and Lesbian Rights in Portugal and in Mexico. Journal of Homosexuality, 00(00). 1-18.

Crawford. I.. McLeod. A.. Zamboni. B. \& Jordan. M. (1999). Psychologists' attitudes toward gay and lesbian parenting. Professional Psychology: Research and Practice. 30(4). 394-401.

Cullinan. M. (2016). Thousands of Colombians join nationwide protest of gender ideology in public schools. Retrieved June 26. 2018. from https://www.lifesitenews.com/news/thousands-of-colombians-joinnationwide-protest-of-gender-ideology-in-publi

Frías-Navarro. D. (2009). Scale of beliefs about children's adjustment in same-sex families.

Frias-Navarro. D.. Monterde-i-Bort. H.. Pascual-Soler. M.. \& Badenes-Ribera. L. (2015). Etiology of homosexuality and attitudes toward same-sex parenting: A randomized study. Journal of Sex Research. 52(2). 151-161.

Glass. V.. Willox. L.. Barrow. K. \& Jones. S. (2015). Struggling to move beyond acknowledgment: Celebrating gay and lesbian families in preschool environments. Journal of GLBT Family Studies, 12(3). 217-241.

Gusberti. J.. Klaime. S.. Kaufert. T. \& Silva. D. (2019). Pesquisa de opinião sobre adoção homoafetiva no Brasil Brazilian Journal of Health Review. 2(4). 3518-3532.

Hamre. B. \& Pianta. R. (2001). Early teacher-child relationships and the trajectory of children's school outcomes through eighth grade. Child Development. 72(2). 625-638.

Hegde. A.. Averett. P.. Parker White. C. \& Deese. S. (2014). Examining preschool teachers' attitudes. comfort. action orientation and preparation to work with children reared by gay and lesbian Parents. Early Child Development and Care. 184(7). 963-976.

Herrera. N. (2014). Las pruebas de Sergio. Retrieved June 26. 2018. from https://www.elespectador.com/noticias/bogota/pruebas-de-sergio-articulo-515085

Hirst. M.. Jervis. N.. Visagie. K.. Sojo. V.. \& Cavanagh. S. (2011). Transition to primary school: A review of the literature. Canberra: Commonwealth of Australia.

Kintner-Duffy. V.. Vardell. R.. Lower. J. \& Cassidy. D. (2012). "The changers and the changed": Preparing early childhood teachers to work with lesbian. gay. bisexual. and transgender families. Journal of Early Childhood Teacher Education. 33(3). 208-223.

Kosciw. J. \& Diaz. E. (2008). Involved, invisible. ignored: The experiences of lesbian. gay, bisexual and transgender parents and their children in our nation's K-12 schools. New York: Gay. Lesbian. Straight Education Network. 
Larrabee. T. \& Kim. Y. (2010). Preservice Elementary Teachers' Perceptions of Family: Considering Future Instruction on Lesbian- and Gay-Headed Families. Journal of Research in Childhood Education. 24(4). 351-365.

Lindsay. J.. Perlesz. A.. Brown. R.. Mcnair. R.. Vaus. D. de. \& Pitts. M. (2006). Stigma or Respect: Lesbian-parented Families Negotiating School Settings. Sociology. 40(6). 1059-1077.

Lubbe. (2013). LGBT parents and their children: Non-western research and perspectives. In A. E. Goldberg \& K. R. Allen (Eds.). Handbook of LGBT-parent families: Innovations in research and implications for practice2 (pp. 209-224). Springer Science+Business Media.

Mercier. L. R.. \& Harold. R. D. (2003). At the interface: Lesbianparent families and their children's schools. Children \& Schools. 25(1). 35-47.

Ministerio de Educación Nacional. (2014). Sentido de la educación inicial. Bogotá: Ministerio de Educación Nacional. Retrieved from http:/www.colombiaaprende.edu.co/primerainfancia

Ministerio de Salud. \& Profamilia. (2017). Encuesta Nacional de Demografía y Salud (ENDS) 2015 - Tomo 2: Componente de Salud Sexual y Reproductiva. Retrieved from http://dhsprogram.com/pubs/pdf/FR334/ FR334.2.pdf

Newton. S. \& Chonody. J. (2014). Heterosexual Attitudes Toward Same-Sex Marriage: The Influence of Attitudes Toward Same-Sex Parenting. Journal of GLBT Family Studies, 10(4), 404-421.

Pennington. J. \& Knight. T. (2011). Through the lens of hetero-normative assumptions: re-thinking attitudes towards gay parenting. Culture. Health \& Sexuality. 13(1). 59-72.

Ruiz. S. (2013). Familias homoparentales en España: integración social. necesidades y derechos. España: Universidad Autonoma de Madrid.

Souto-Manning. M. \& Hermann-Wilmarth. J. (2008). Teacher inquiries into gay and lesbian families in early childhood classrooms. Journal of Early Childhood Research. 6(3). 263-280.

Vargas. E. (2013). Sexualidad... mucho más que sexo. Bogotá: Ediciones Uniandes.

Vargas. E.. Ripoll. K.. Carrillo. S.. Rueda Sáenz. M.. \& Castro. J. (2011). Experiencias familiares de padres y madres con orientaciones sexuales diversas: Aportes de la investigación. Bogotá: Ediciones Uniandes.

Ventura-León. J. \& Caycho. T. (2017). Q de Cohen: Comparación de Correlaciones entre Muestras Independientes en base a Urzúa et al. Rev Med Chile. 145. 410-412.

Weiner. B. \& Zinner. L. (2015). Attitudes Toward Straight. Gay Male. and Transsexual Parenting. Journal of Homosexuality. 62(3). 327-339.

White. L. (2015). Impact of children's literature on family structures and children's perceptions of family. St. John Fisher College. 